\title{
Effect of irradiance data on the optimal sizing of photovoltaic water pumping systems
}

\author{
Simon Meunier ${ }^{1, *}$, Loic Queval ${ }^{1}$, Matthias Heinrich ${ }^{2}$, Elvire A. de la Fresnaye ${ }^{3}$, Judith A. Cherni ${ }^{3}$, Lionel \\ Vido $^{4}$, Arouna Darga ${ }^{1}$, Philippe Dessante ${ }^{1}$, Bernard Multon ${ }^{4}$, Peter K. Kitanidis ${ }^{5}$, Claude Marchand ${ }^{1}$ \\ ${ }^{1}$ GeePs | Group of electrical engineering - Paris, CNRS, CentraleSupélec, Univ. Paris-Sud, Univ. Paris- \\ Saclay, Sorbonne Univ., Gif-sur-Yvette, France \\ ${ }^{2}$ DargaTech SARL, Ouagadougou, Burkina Faso \\ ${ }^{3}$ Centre for Environmental Policy, Imperial College London, London, UK \\ ${ }^{4}$ SATIE, CNRS, Univ. Cergy-Pontoise, ENS Rennes, Univ. Rennes, Cergy-Pontoise, France \\ ${ }^{5}$ Department of Civil and Environmental Engineering, Stanford Univ., Stanford, USA \\ *Correspondence: simon.meunier@centralesupelec.fr
}

\begin{abstract}
Photovoltaic water pumping systems (PVWPS) are an interesting solution to improve water access in off-grid areas. Irradiance being the main input of PVWPS models, the source (local sensor or satellite database) and temporal resolution of irradiance data strongly influence the accuracy of PVWPS models and the optimal sizing obtained from these models. We show that we can use satellite data instead of data from a local sensor and a temporal resolution of 1 hour without significantly changing the model accuracy and optimization results. These results can allow to intensify the implementation of PVWPS in off-grid areas.
\end{abstract}

Keywords - photovoltaic system, water pumping, experimental data, optimization, life-cycle cost, solar resource

\section{INTRODUCTION}

Water pumping systems powered by photovoltaic energy have proved to be an interesting solution to improve water access in off-grid areas of developing countries $[1,2]$. Several models of photovoltaic water pumping systems (PVWPS) were developed. These models use input irradiance data from local sensors [3, 4] or satellite databases [5, 6]. Moreover, the temporal resolution of the irradiance data varies from 1 minute [7] up to 1 day [8]. To the best knowledge of the authors, the choice of the data source (sensor or satellite) and of the temporal resolution is arbitrary in existing models. In addition, no study has assessed the influence of these factors on PVWPS models output and PVWPS optimal sizing.

In this article, we evaluate the impact of the data source (sensor or satellite) and of the temporal resolution of irradiance data on PVWPS model accuracy and on the optimal sizing of these systems. Firstly, we show to which extent satellite data can be used instead of data from a local sensor. This can favor the implementation of PVWPS in areas where no local irradiance measurements are available. Secondly, we determine the coarsest temporal resolution that can be used for monitoring and sizing PVWPS for domestic water access. This can decrease the data collection cost, and reduce the computing time for the optimization.
The model of the PVWPS is detailed in section II. The details about the source (sensor or satellite) and the temporal resolution of irradiance data are presented in section III. The methodology is described in section IV. The effects of the source and of the temporal resolution of irradiance data on model accuracy and on optimal sizing are presented in sections V and VI respectively.

\section{Photovoltaic Water Pumping System Model}

We consider PVWPS with the architecture shown in Fig. 1. Such systems are common for providing water for domestic use. The controller regulates the energy provided by the photovoltaic (PV) modules to the motor-pump, according to the water level in the tank $[9,10]$. The inhabitants collect water at the fountain.

We have developed a PVWPS model adapted to such systems [10]. A block diagram of the model is presented in Fig. 2. The model inputs are the irradiance on the plane of the PV modules $G_{p v}$, the ambient temperature $T_{a}$ and the water collection flow rate at the fountain $Q_{c}$. The output of the model is the water level in the tank $H_{t k}$.

The model has been validated by using data acquired on a PVWPS which provides water to 250 inhabitants in the village of Gogma in Burkina Faso, sub-Saharan Africa (Fig. 3) [10]. In this PVWPS, there are $620 \mathrm{~W}_{\mathrm{p}}$ of PV modules, a motor-pump SQFlex 5A-7 from Grundfos [11] and a tank of $11.4 \mathrm{~m}^{3}$. A video of the village and of the PVWPS is available at the following link https://youtu.be/VrjM0edKVsI. The quantities that have been measured are specified in Fig. 2. They have been acquired with a time step of $\sim 2.2 \mathrm{~s}$ since January 2018 . The data were rescaled to an evenly spaced temporal resolution of 1 minute by nearest interpolation. The evolution of the measured quantities has been reported in [10] and [12].

In this article, only the source and the temporal resolution of $G_{p v}$ change (see section III) while we use measured data with a 1 minute temporal resolution for the two other inputs of the model $\left(T_{a}\right.$ and $\left.Q_{c}\right)$. Indeed, we showed that $T_{a}$ has a small 
effect on the model output [12] and we investigated the influence of $Q_{c}$ in a previous article [13].

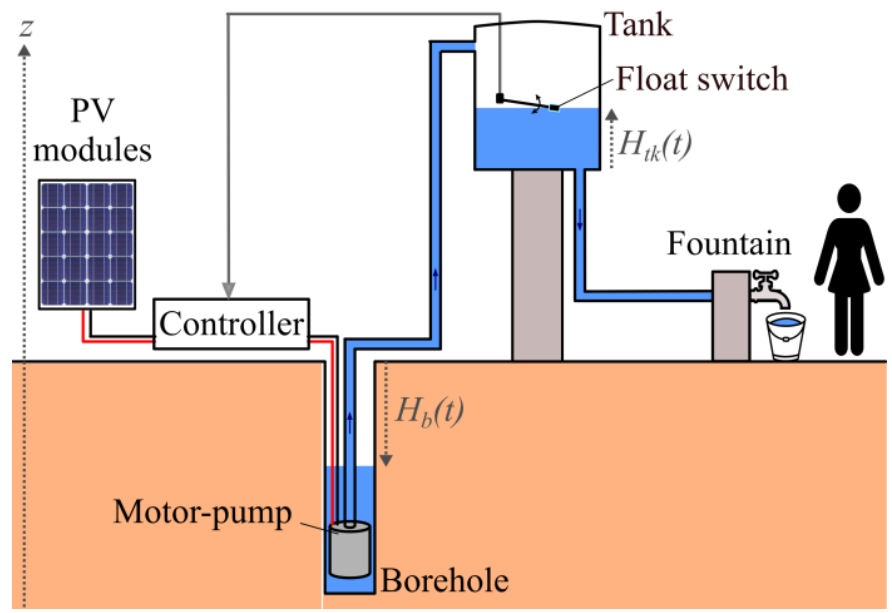

Fig. 1 - Architecture of the photovoltaic water pumping system. $H_{b}$ : water level in the borehole $\left(H_{b}<0\right) ; H_{t k}$ : water level in the tank.

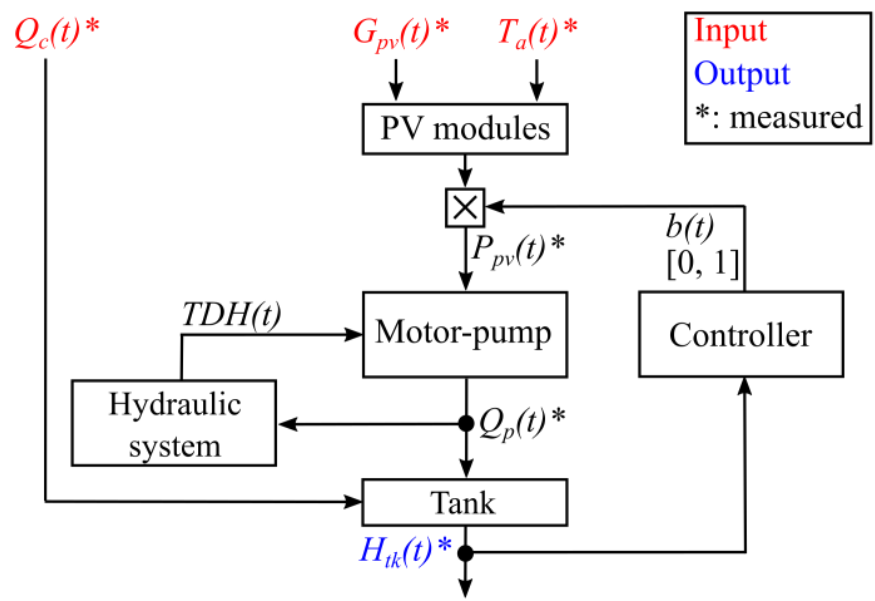

Fig. 2 - Block diagram of the PVWPS model. $Q_{c}$ : water collected flow rate, $G_{p v}$ : irradiance on the plane of the PV modules, $T_{a}$ : ambient temperature, $P_{p v}$ : input power to the motor-pump, $Q_{p}$ : pumped flow rate, TDH: total dynamic head, $H_{t k}$ : water level in the tank, $b$ : triggering signal from the controller, $t$ : time.

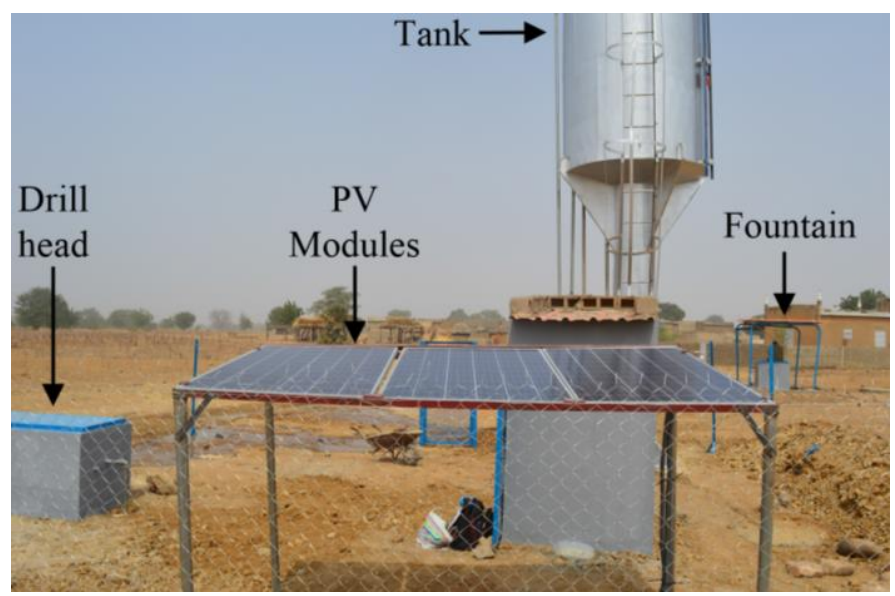

Fig. 3 - Test site: photovoltaic water pumping system for 250 inhabitants of the village of Gogma in rural Burkina Faso.

\section{SOURCE AND TEMPORAL RESOLUTION OF IRRADIANCE DATA}

\section{A. Data Source: Local Sensor or Satellite Data}

The irradiance on the plane of the PV modules $G_{p v}$ is measured at our test site with a RG100 sensor from SOLEMS, which is a calibrated photovoltaic cell [14].

The satellite irradiance data were obtained from the Copernicus Atmosphere Monitoring Service which provides the direct normal irradiance $G_{d n}$, the global horizontal one $G_{g h}$ and the diffuse horizontal one $G_{d h}$ with a temporal resolution of 1 minute [15]. In order to provide data with a 1 minute temporal resolution, the Copernicus Atmosphere Monitoring Service performs the interpolation of satellite images, which are taken every 15 minutes [15]. The irradiance on the plane of the PV modules $G_{p v}$ is then deduced by [16]:

$$
\begin{gathered}
G_{p v}(t)=G_{d n}(t) \cos (A O I(t, \theta, \alpha))+G_{g h}(t) \rho \frac{1-\cos (\theta)}{2} \\
+G_{d h}(t) \frac{1+\cos (\theta)}{2}
\end{gathered}
$$

where $t$ is the time, $\rho$ is the albedo of the surrounding environment, $\theta$ and $\alpha$ are the tilt and azimuth of the PV modules respectively, $A O I$ is the angle of incidence between the sun's rays and the PV modules. For the PVWPS of Gogma, $\rho=0.25, \theta=11^{\circ}$ and $\alpha=180^{\circ}$. The $A O I$ is computed by using the MATLAB toolbox PVLIB developed by the Sandia National Laboratories [17].

Fig. 4 shows the irradiance on the plane of the PV modules $G_{p v}$ from both the local sensor and satellite data with a temporal resolution $\tau$ of 1 minute for one given day of our dataset. Data acquired by the local sensor with a temporal resolution of 1 minute are the most accurate irradiance data.

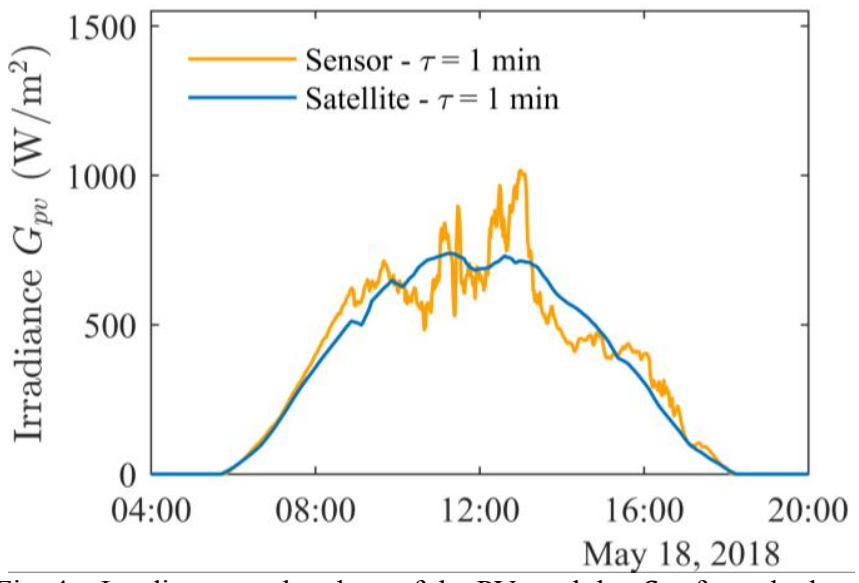

Fig. 4 - Irradiance on the plane of the PV modules $G_{p v}$ from the local sensor and from satellite data.

\section{B. Temporal Resolution}

Data with a given temporal resolution $\tau$ can be obtained with two different methods: sampling and averaging. The properties of sampling and averaging are compared in Table 1. 
Table 1 -Comparison between methods for setting the temporal resolution $\tau$ : sampling and averaging

\begin{tabular}{|l|l|l|}
\hline & Sampling & Averaging \\
\hline Principle & Data directly acquired at a time step $\tau$. & $\begin{array}{l}\text { Data acquired at a time step } \tau_{1}(<\tau) \text { and then } \\
\text { averaged over the interval } \tau .\end{array}$ \\
\hline $\begin{array}{l}\text { Influence on daily integral } \\
\text { of irradiance } \boldsymbol{I}\left(\boldsymbol{G}_{\boldsymbol{p} v}\right)\end{array}$ & Two profiles with a different $\tau$ have a different $I\left(G_{p v}\right)$. & $\begin{array}{l}\text { Two profiles with a different } \tau \text { have the same } \\
I\left(G_{p v}\right) .\end{array}$ \\
\hline $\begin{array}{l}\text { Influence on amount of } \\
\text { collected data }\end{array}$ & $\begin{array}{l}\text { Choosing a large value for } \tau \text { decreases the amount of } \\
\text { collected data. }\end{array}$ & $\begin{array}{l}\text { Choosing a large value for } \tau, \text { with the same } \tau_{1}, \\
\text { does not change the amount of collected data. }\end{array}$ \\
\hline Main application & $\begin{array}{l}\text { Choosing a large value for } \tau \text { allows to cut down data } \\
\text { collection cost by using sensors with lower temporal } \\
\text { performances, decreasing the energy consumption of the } \\
\text { data logger and reducing the amount of data stored and } \\
\text { transmitted. }\end{array}$ & $\begin{array}{l}\text { Increasing } \tau \text { allows to decrease computation } \\
\text { time for simulating and therefore optimizing } \\
\text { the system. }\end{array}$ \\
\hline
\end{tabular}

In order to build the profile for sampling at a temporal resolution $\tau$ from the 1 minute sensor or satellite data (cf Fig. 4), we replace all the values of $G_{p v}$ in a time interval $\tau$ by the value of $G_{p v}$ at the middle of this interval. For averaging, we replace all the values of $G_{p v}$ in a time interval $\tau$ by the average of these values. Fig. 5 presents the profiles obtained by sampling and averaging from the sensor data for a temporal resolution $\tau$ of 2 hours. We notably observe that, from 12:00 to 14:00, the values of $G_{p v}$ for sampling and averaging are very different. The daily integral of the reference profile with a 1 minute temporal resolution (in orange in Fig. 5) is equal to $5.5 \mathrm{kWh} / \mathrm{m}^{2} /$ day. As expected, this value is equal to the integral of the profile obtained by averaging (in red) and it is different from the integral of the profile obtained by sampling (in pink), which is $5.9 \mathrm{kWh} / / \mathrm{m}^{2} /$ day.

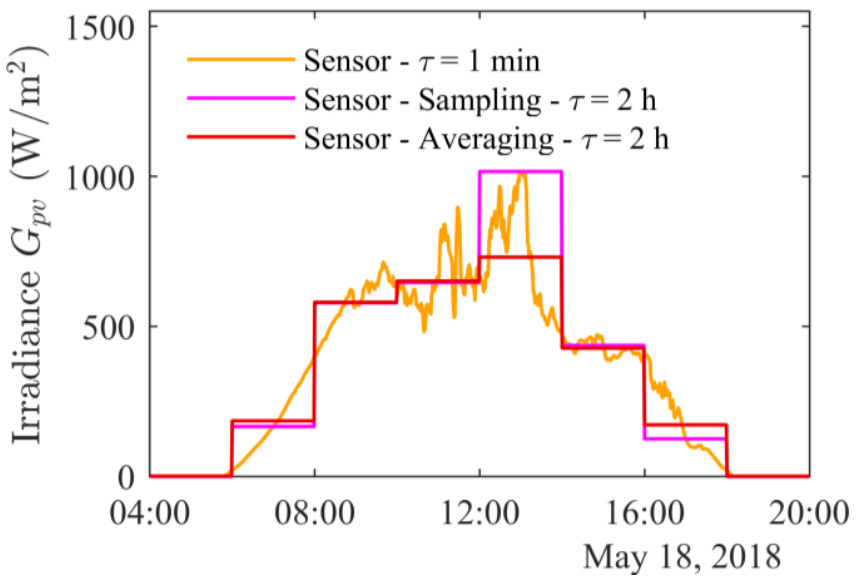

Fig. 5 - Profiles obtained by averaging and sampling from the sensor data for a temporal resolution $\tau$ of 2 hours.

\section{METHODOLOGY}

The analysis aims at determining the influence of the source and of the temporal resolution of irradiance data on the PVWPS model accuracy and on the optimal sizing of PVWPS. For each data source (sensor or satellite) we consider both averaging and sampling. In addition, we consider 15 values of the temporal resolution $\tau$ ranging from 1 minute to 1 day. Fig. 6 summarizes the possible combinations and shows an example of combination in red. In total there are $2 \times 2 \times 15=60$ combinations.

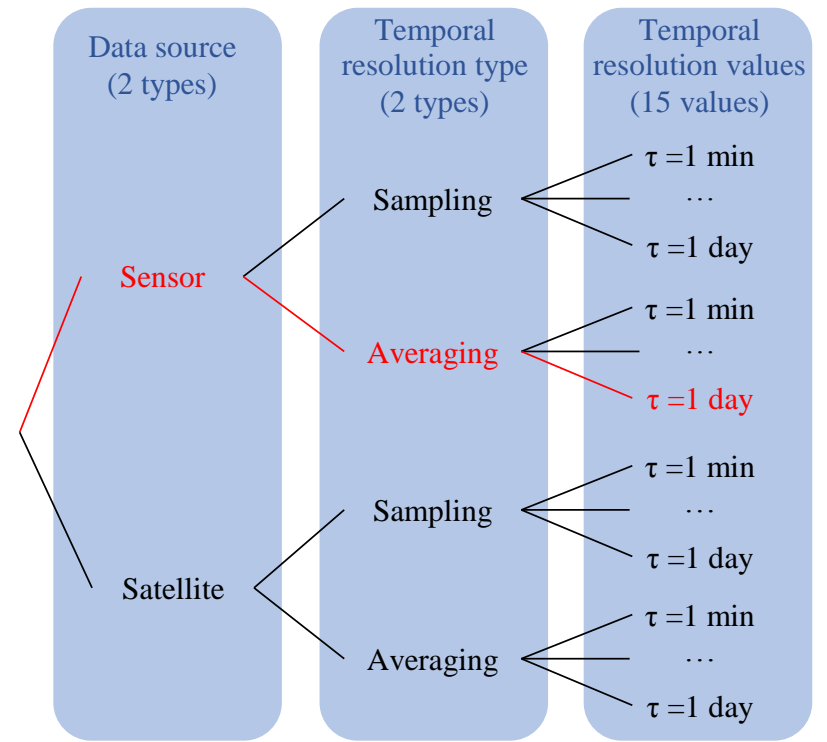

Fig. 6 - Possible combinations between irradiance data source, temporal resolution type and value. A possible combination is in red.

We consider two periods of two weeks. The first period starts on the $16^{\text {th }}$ of May and ends on the $29^{\text {th }}$ of May 2018 and it represents the dry season. The second one starts on the $29^{\text {th }}$ of July and ends on the $11^{\text {th }}$ of August 2018 and it represents the wet season. The daily water collection at the PVWPS is significantly lower during the wet season $\left(\sim 5 \mathrm{~m}^{3} /\right.$ day $)$ than during the dry one $\left(\sim 10 \mathrm{~m}^{3} /\right.$ day $)$. The average daily integral of the irradiance during the wet and dry seasons are nearly the same $\left(\sim 5.5 \mathrm{kWh} / \mathrm{m}^{2} /\right.$ day $)$ but the temporal variability of the irradiance is higher during the wet season, due to the presence of more clouds.

\section{INFLUENCE ON MODEL ACCURACY}

In this section, we assess the effect of the source (sensor or satellite) and temporal resolution of irradiance data on PVWPS model accuracy. For each season (dry or wet) and for each combination presented in Fig. 6, we:

1) simulate the water level in the tank from the PVWPS model during the considered two-week period $H_{t k}^{\text {sim,comb }}$.

2) compute the normalized root mean square error NRMSE between the simulated and measured water level in the tank: 


$$
\text { NRMSE }=\frac{1}{H_{t k, c}} \sqrt{\frac{\sum_{i=1}^{n}\left(H_{t k}^{\text {sim,comb }}(i)-H_{t k}^{\text {meas }}(i)\right)^{2}}{n}}
$$

where $H_{t k, c}$ is the height of the tank, $H_{t k}^{\text {meas }}$ is the measured water level, $n$ is the number of points in the simulation period.

Fig. 7 and Fig. 8 present the results obtained for the dry season and the wet season respectively.

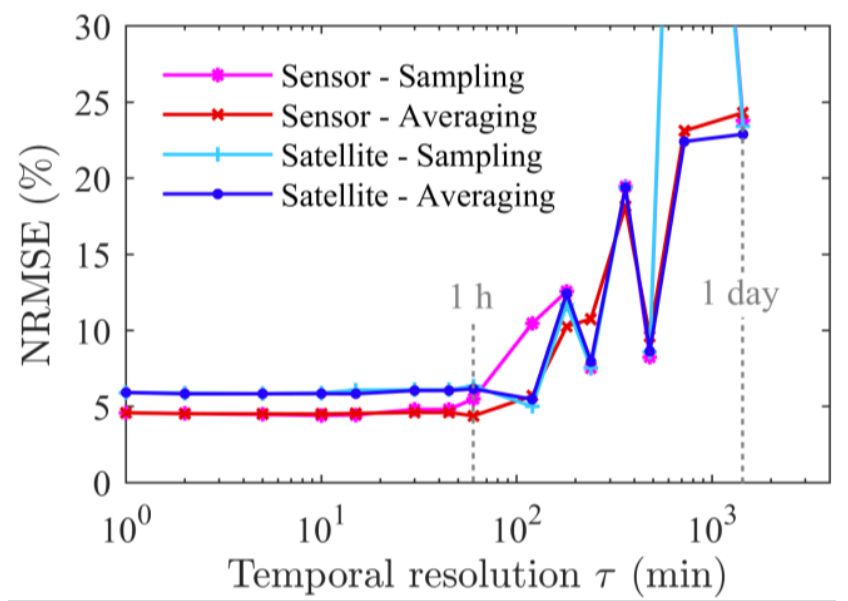

Fig. 7 - NRMSE on the water level in the tank for the different irradiance data sources and temporal resolutions - dry season. The two points that are out of the frame are: NRMSE(SensorSampling, $\tau=12 \mathrm{~h}$ ) $=74 \%$ and NRMSE(Satellite-Sampling, $\tau=12 \mathrm{~h}$ ) $=74 \%$.

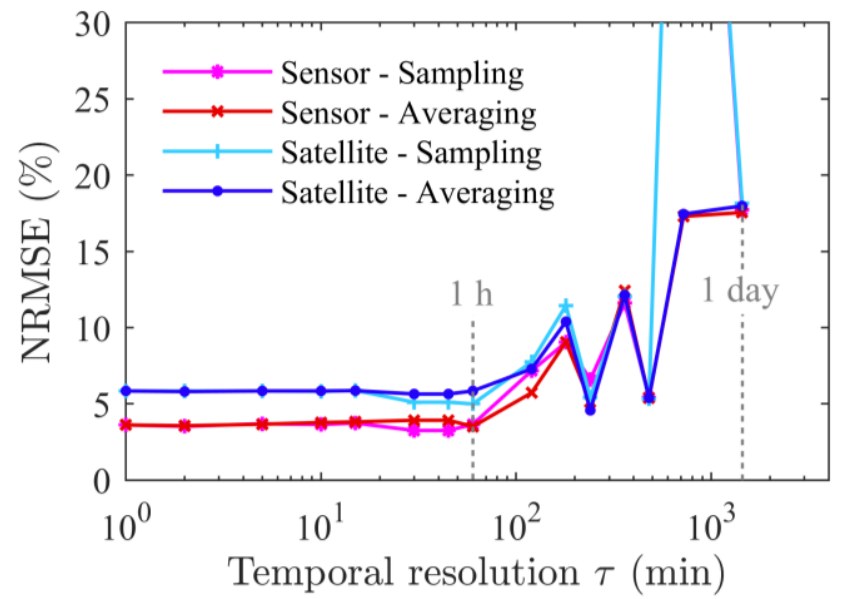

Fig. 8 - NRMSE on the water level in the tank for the different irradiance data sources and temporal resolutions - wet season. The two points that are out of the frame are: NRMSE(SensorSampling, $\tau=12 \mathrm{~h}$ ) $=77 \%$ and NRMSE(Satellite-Sampling, $\tau=12 \mathrm{~h}$ ) $=77 \%$.

We observe that, for values of $\tau$ smaller than $1 \mathrm{~h}$, the temporal resolution does not have a significant impact on the model accuracy. Moreover, for these values of $\tau$, results with sampling and averaging are similar and using satellite data instead of data from the local sensor decreases model accuracy by only 1 to $2 \%$. Results also indicate that using values of $\tau$ larger than 1 hour strongly decreases the model accuracy. We notably observe very high values of the NRMSE ( $>70 \%$ ) for sampling with a temporal resolution $\tau$ of 12 hours both for sensor and satellite data. Indeed, for $\tau=12 \mathrm{~h}$, the only points of the irradiance curve that are selected are the irradiance at 6:00 and at 18:00, which are very close to $0 \mathrm{~W} / \mathrm{m}^{2}$ (cf Fig. 4). Consequently, the simulated pumped flow rate is close to $0 \mathrm{~m}^{3} / \mathrm{s}$ at every instant, and the simulated water level in the tank $H_{t k}^{s i m, c o m b}$ is nearly at $0 \mathrm{~m}$ during the whole simulation period, therefore explaining the large NRMSE. This illustrates the limitations of sampling for values of $\tau$ of several hours.

\section{INFLUENCE ON OPTIMAL SYSTEM SIZING}

In this section, we examine the effect of the source (sensor or satellite) and temporal resolution of irradiance data on the PVWPS optimal sizing.

\section{A. Optimization Problem [12]}

The peak power of the PV modules $P_{p v, p}$, the motor-pump reference $M P_{i}$ and the tank volume $V_{t k}$ are the variables of the optimization. The characteristic curves of 8 references of submersible SQFlex motor-pumps from Grundfos were digitized (references 8A-3, 5A-3, 0.6-2, 1.2-2, 11A-3, 8A-5, $5 \mathrm{~A}-7,2.5-2)$ [18]. The objective function is the life-cycle cost $L C C$ of the PVWPS in $\mathrm{k} \$[19]$ :

$$
L C C=C A P E X+\sum_{j=1}^{L} \frac{O P E X(j)}{(1+r)^{j}}
$$

where the capital expenditure $C A P E X$ is given by:

$$
C A P E X=0.00085 P_{p v, p}+C\left(M P_{i}\right)+0.76 V_{t k}
$$

and the operational expenditure $O P E X$ is given by:

$$
\operatorname{OPEX}(j)=\left\{\begin{array}{c}
\frac{C A P E X}{100} \text { if } j \neq 10 \\
\frac{C A P E X}{100}+C\left(M P_{i}\right) \text { if } j=10
\end{array}\right.
$$

where $r$ is the discount rate, $L$ is the lifetime of the PVWPS, $C\left(M P_{i}\right)$ is the cost of the motor-pump reference $M P_{i}$. We choose $L=20$ years and $r=5.6 \%$ [19]. The values of $C\left(M P_{i}\right)$ are provided in [18] and the coefficients in equations (3) and (4) were determined from local cost data from Burkina Faso. The motor-pump is replaced after 10 years (cf eq. (4)).

There are three constraints of the optimization. Firstly, the water level in the tank $H_{t k}$ (Fig. 1) has to remain higher than $0 \mathrm{~m}$. This allows to meet the water demand of the users. Secondly, the water level in the borehole $H_{b}$ (Fig. 1) has to remain higher than $-20 \mathrm{~m}$. This prevents dewatering and the motor-pump from running dry. Thirdly, for each motor-pump reference $M P_{i}$, the total dynamic head $T D H$ has to remain smaller than the maximum pumping height of the motor-pump, which is given in the motor-pump datasheet. The PVWPS model is needed to verify these constraints. As a consequence, it is at this stage that the source and the temporal resolution of irradiance data have an influence. A differential evolution algorithm is used for this optimization [20]. 


\section{B. Influence of Irradiance Data}

For each season (dry or wet) and for each combination presented in Fig. 6, we perform the optimization. Fig. 9 presents the optimal life-cycle cost $L C C$ for each combination for the dry season. Results for the wet season are shown in Fig. 10.

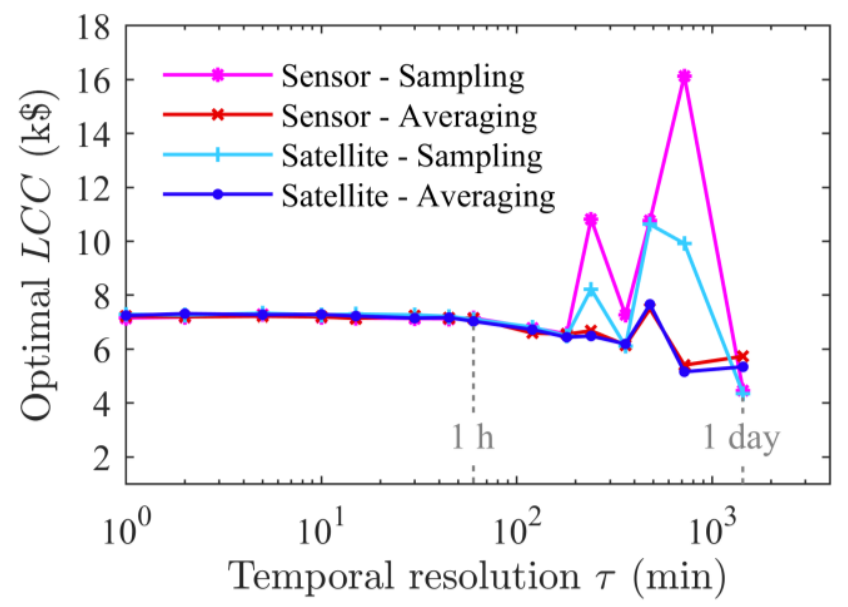

Fig. 9 - PVWPS optimal sizing for the different irradiance data sources and temporal resolutions - dry season.

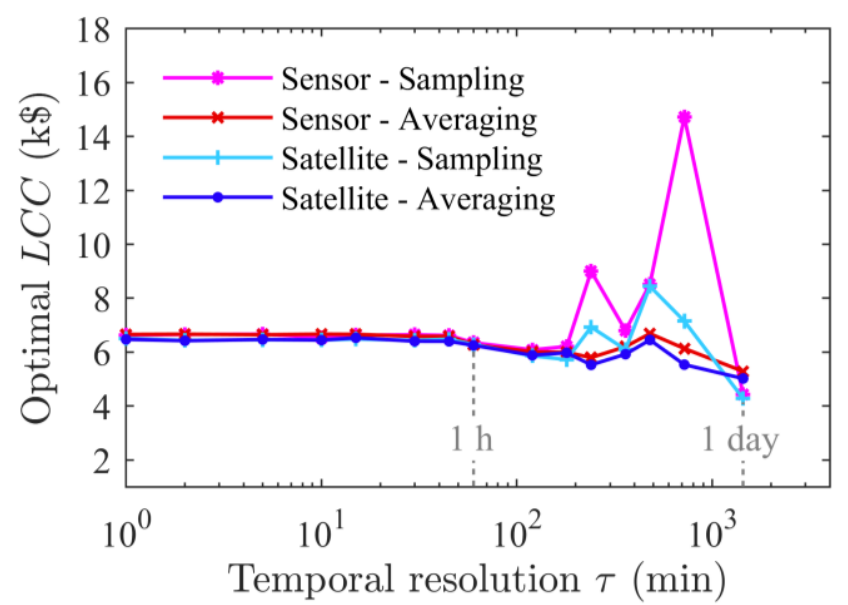

Fig. 10 - PVWPS optimal sizing for the different irradiance data sources and temporal resolutions - wet season.

The reference optimal $L C C$, and therefore the reference optimal system sizing, is the one obtained for the sensor irradiance data with a 1 minute temporal resolution. The higher the variation from the reference optimal $L C C$, the higher the error on the optimal system sizing. For values of $\tau$ smaller than 1 hour, we observe that the temporal resolution $\tau$ does not have a significant impact on the optimal $L C C$ and that the optimal $L C C$ is close to the reference optimal $L C C$. Besides, for these values of $\tau$, results for sampling and averaging are similar and using satellite data instead of data from the local sensor has a small impact on the optimal LCC. Finally, for values of $\tau$ larger than 1 hour, the temporal resolution $\tau$ has a significant impact on the optimal $L C C$, and the optimal $L C C$ obtained differ significantly from the reference optimal $L C C$.

\section{CONCLUSION AND RECOMMENDATIONS}

The results indicate that hourly irradiance data can be used instead of data with a smaller value of $\tau$ (e.g. 1 minute), without significantly modifying the model accuracy nor the optimal sizing. In addition, obtaining these hourly data through sampling or averaging has nearly no influence on the results. Finally, for hourly data, the results on model accuracy and optimal sizing are similar for sensor and satellite data.

These results may be of interest to non-governmental organizations and companies which size and/or monitor PVWPS for domestic water supply. Depending on the installation phase in which they are (optimal system sizing or site monitoring) we propose them to use the following irradiance data:

1) Optimal system sizing (before installation): satellite data obtained by averaging with a 1 hour temporal resolution. When sizing a PVWPS, one rarely has access to long term local irradiance measurements at the future installation site. The results of this study, and notably the ones on optimal sizing in section VI, show that using satellite data is a viable alternative. In addition, for satellite data, averaged data are directly provided in the databases [15]. Therefore, averaged data should be prioritized as they do not change the daily integral of the irradiance, contrarily to data obtained by sampling (see section III). Besides, as shown in section VI, irradiance data with a temporal resolution of 1 hour can be used for optimal sizing. This notably permits to decrease computing time for the optimization of PVWPS.

2) Site monitoring (after installation): data from local sensor obtained by sampling with a 1 hour temporal resolution. If the installation is being monitored, we recommend to connect an irradiance sensor to the existing data logger. Furthermore, as shown by this study, and notably the results on model accuracy in section $\mathrm{V}$, acquiring irradiance data with a time step of 1 hour (sampling - 1 hour) is sufficient for monitoring. This therefore allows to use a sensor with a sampling rate of up to 1 hour, to decrease the energy consumption of the data logger and to reduce the amount of data stored and transmitted through internet or the telephone network, therefore cutting down monitoring costs.

Finally, an analogy can be made between PVWPS with water tank storage, as the one presented here, and photovoltaic systems with battery storage such as solar home systems. The methodology presented in this article could therefore also be used to study the effect of the source and the temporal resolution of irradiance data on the optimal sizing of photovoltaic systems with battery storage.

\section{ACKNOWLEDGMENT}

This work is supported by a public grant overseen by the French National research Agency (ANR) as part of the "Investissement d'Avenir" program, through the "IDI 2015" project funded by the IDEX Paris-Saclay, ANR-11-IDEX000302. 


\section{REFERENCES}

[1] S. S. Chandel, M. Nagaraju Naik, and R. Chandel, "Review of solar photovoltaic water pumping system technology for irrigation and community drinking water supplies," Renewable \& Sustainable Energy Reviews, vol. 49, pp. 1084-1099, 2015.

[2] S. Meunier, D. T. Manning, L. Queval, J. A. Cherni, P. Dessante, and D. Zimmerle, "Determinants of the marginal willingness to pay for improved domestic water and irrigation in partially electrified Rwandan villages," to be published in International Journal of Sustainable Development \& World Ecology, 2019.

[3] P. Sadasivam, M. Kumaravel, K. Vasudevan, and A. Jhunjhunwala, "Analysis of subsystems behaviour and performance evaluation of solar photovoltaic powered water pumping system," in 39th IEEE Photovoltaic Specialists Conference (PVSC), 2013, pp. 2932-2937.

[4] D. H. Muhsen, A. B. Ghazali, T. Khatib, I. A. Abed, E. M. Natsheh "Sizing of a standalone photovoltaic water pumping system using a multi-objective evolutionary algorithm," Energy, vol. 109, pp. 961-973, 2016.

[5] P. E. Campana, H. Li, and J. Yan, "Dynamic modelling of a PV pumping system with special consideration on water demand," Applied Energy, vol. 112, pp. 635-645, 2013.

[6] A. Allouhi, M.S. Buker, H. El-houari, A. Boharb, M. Benzakour Amine, T. Kouskou, A. Jamil "PV water pumping systems for domestic uses in remote areas: sizing process, simulation and economic evaluation," Renewable Energy, vol. 132, pp. 798-812, 2019.

[7] J. K. Kaldellis, G. C. Spyropoulos, K. A. Kavadias, and I. P. Koronaki, "Experimental validation of autonomous PV-based water pumping system optimum sizing," Renewable Energy, vol. 34, no. 4, pp. 11061113, 2009.

[8] Z. Girma, "Techno-economic analysis of photovoltaic pumping system for rural water supply in Ethiopia," International Journal of Sustainable Energy, vol. 36, no. 3, pp. 277-295, 2017.

[9] S. Meunier, M. Heinrich, J. A. Cherni, L. Queval, P. Dessante, L. Vido, A. Darga, B. Multon and C. Marchand, "Modélisation et validation expérimentale d'un système de pompage photovoltaïque dans une communauté rurale isolée du Burkina Faso," in Symposium de Génie Electrique (SGE), 2018.
[10] S. Meunier, M. Heinrich, L. Queval, J. A. Cherni, L. Vido, A. Darga, P. Dessante, B. Multon, P. K. Kitanidis and C. Marchand, "A validated model of a photovoltaic water pumping system for off-grid rural communities," Applied Energy, vol. 241, pp. 580-591, May 2019.

[11] Grundfos, "Performance curve of SQFlex 5A-7 motor-pump," 2019. [Online]. Available:

https://product-selection.grundfos.com/product-detail.productdetail.html?custid $=$ GMA\&productnumber $=95027342 \& q$ cid $=34276834$ $\underline{2}$

[12] S. Meunier, L. Queval, A. Darga, P. Dessante, C. Marchand, M. Heinrich, J. Cherni, E. A. de la Fresnaye, L. Vido, B. Multon and P. K. Kitanidis, "Modelling and optimal sizing of photovoltaic water pumping systems Sensitivity analysis," in International Conference on Ecological Vehicles and Renewable Energies (EVER), 2019.

[13] S. Meunier, L. Queval, A. Darga, P. Dessante, C. Marchand, M. Heinrich, J. Cherni, L. Vido and B. Multon "Influence of the temporal resolution of the water consumption profile on photovoltaic water pumping systems modelling and sizing," in 7th International Conference on Renewable Energy Research and Applications (ICRERA), 2018, p. 494-499.

[14] Solems, "RG solar radiation sensors," 2016. [Online]. Available: https://www.solems.com/wpcontent/uploads/Solar radiation sensors RG.pdf

[15] Copernicus Atmosphere Monitoring Service, "User’s Guide,” 2018. [Online]. Available: $\mathrm{http}: / / \mathrm{www} \cdot$ sodapro.com/documents/10157/326332/CAMS72_2015SC3_D72.1.3.1_201 8 UserGuide v1 201812.pdf/95ca8325-71f6-49ea-b5a6-8ae4557242bd

[16] L. M. Fraas and L. D. Partain, Solar cells and their applications, $2^{\text {nd }} e d$, Hoboken, NJ, USA: John Wiley \& Sons, Inc., 2010

[17] Sandia National Laboratories, "PV_LIB Toolbox;" 2018. [Online]. Available: https://pvpmc.sandia.gov/applications/pv_lib-toolbox/

[18] Off-grid Europe, "Grundfos SQ Flex." 2017. [Online]. Available: https://www.off-grid-europe.com/accessories/pumps/grundfos/sq-flex

[19] E. André de La Fresnaye, "A financial and technical assessment of solar versus hand water pumping for off-grid area - the case of Burkina Faso," MSc Thesis, Imperial College London, UK, 2018.

[20] K. Price, R. M. Storn, and J. A. Lampinen, Differential evolution: a practical approach to global optimization (natural computing series), Secaucus, NJ, USA: Springer-Verlag New York, Inc., 2005. 